\title{
Enhancement of Small Signal Stability of SMIB System with Robust Power System Stabilizer
}

\author{
R. Ramya, Soumyadeep Maity
}

\begin{abstract}
As power system experiences low frequency oscillations due to disturbances, these low frequency oscillations are related to the small signal stability of a power system. The phenomenon of stability of synchronous machine under small perturbations is explored by examining the case of an SMIB system. The analysis of SMIB gives physical insight into the problem of low frequency oscillations. The SMIB system is predominant in local mode low frequency oscillations. These oscillations may sustain and grow to cause system separation if no adequate damping is available. The damping is provided by adding Power System Stabilizer for Synchronous Machine. In addition, as power system is nonlinear in nature, application of robust control techniques is mandatory to face the challenge of dynamic conditions. Hence, this work aims to design robust Power System Stabilizer for Synchronous Machine in order to damp the rotor speed deviations.
\end{abstract}

Keywords - Power system stabilizer, SMIB, Robust control techniques.

\section{INTRODUCTION}

Power system stability is the major concern in the system operation. The results of the instability increase the instability of the system. Any type of disturbance affects the synchronism can be large or small. The duration of the fault has a huge impact on the system stability. The stability of the system can be categorized into small signal and transient stability.

The low frequency oscillations are first detected during $1960-70$ 's. It usually takes a waveform like sine wave, square wave etc. and it can also incorporate with the user defined signals. The oscillations ranging from $0.1-3 \mathrm{~Hz}$ are inter-area and local mode of oscillations. These types of oscillations which led to cause system collapse. The inter-area modes of oscillations caused by the strong linking between the generators of the and tends to oscillates the other generators in the same area. [1]

The local modes of oscillations happen due to one or more synchronous swinging of generators against a large power system. The low frequency oscillations arise due to increment of load in the utility, generation far away from the load and bulk transfer of power that causes generator rotor swings in presence of high gain Automatic Voltage Regulator (AVR). The assumptions of low frequency oscillations are the current and voltage phasors and magnitude varied slowly and the and the shaft of generator mounted rigid for the system. [2]

There are several blackout cases happened across the

R. Ramya, Assistant Professor, EEE, SRM Institute of Science and Technology, Chennai, Tamilnadu, India. (Email: rramyame@gmail.com)

Soumyadeep Maity, PG Student, EEE, SRM Institute of Science and Technology, Chennai, Tamilnadu, India. (Email: soumya.ee95@gmail.com)

world which raised consciousness about the low frequency oscillations. Some of the big incidents of blackouts are:

- United Kingdom (1980), frequency of oscillation of about $0.5 \mathrm{~Hz}$.

- Taiwan $(1984,1989,1990,1991,1992)$ frequency of oscillation of $0.78-1.05 \mathrm{~Hz}$.

- Scandinavia (1997), frequency of oscillation of about $0.5 \mathrm{~Hz}$

- West USA/Canada, System Separation (1996), frequency of oscillation around $1 \mathrm{~Hz}$.

- China Blackout on 6th March (2003), frequency of oscillation about $0.17 \mathrm{~Hz}$.

- Italian Blackout on 28th September (2003), frequency about $0.55 \mathrm{~Hz}$.

The instability arises due to lack of damping torque because of increasing amplitude of rotor oscillations and lack of synchronizing torque due to steady increase of generator rotor angle. The Automatic Voltage Regulator (AVR) provides the negative damping to the system while the Power System Stabilizers (PSS) provide positive damping to the system. The AVR and PSS can prevent the system from the low frequency oscillations. [3]

\section{POWER SYSTEM STABILIZERS}

The stabilization of the power system becomes necessary after the appearance of low frequency oscillations. Power System Stabilizers are used to extent the power transfer limits to wide range by damping the low frequency oscillations. Due to the low time constant voltage regulator, high gain AVR there is a lack of damping torque while transforming the power to the longer distances.

In 1960's stabilizer based on shaft signal introduced on hydraulic units. The delta-omega stabilizers have important considerations regarding it's designs the shaft runout and the minimization of noise. The allowable level is $0.02 \%$ of noise frequency $5 \mathrm{~Hz}$. The stabilizers are limited to the thermal units due to torsional oscillations. After that the delta-p-omega stabilizers are introduced to attenuate higher degree of torsional oscillations. These are used for designing of PSS independent on torsional characteristics for deriving the mechanical power from electrical power signals. This designing is made for multi-purpose. After that integral of power-based stabilizer came into being a filter for single input stabilizers. The frequency-based is based on terminal frequency which is derived from terminal current and 
terminal voltage. Its main advantage is sudden phase shift in transient condition. [4]

The modern stabilizers used in the market are combination of multi-band stabilizers are combination of multi-band and digital stabilizers. The multiband stabilizers are used to handle different oscillation frequencies and has three separate signal bands. The digital stabilizers are produced with excitation systems with complete digital circuitry.

The PSS design categories divided into three categories:

a. Linear Control Methods

b. Non-Linear Methods

c. Empirical Control Methods

The control methodologies are:

a. Classical/Conventional Methods

b. Adaptive and Variable Structure Methods

c. Robust Control Techniques

d. Artificial Intelligence Techniques

e. Digital Control Schemes.

The conventional PSS performance is limited to wide area operating conditions. Nowadays, the robust PSS is used for wider area operations and improve in performances. The robust PSS can maintain the stability of the system under worst case conditions. The settling of robust is much less than conventional PSS as compared. [5]

\section{CONVENTIONAL PSS}

The PSS uses speed change of the generator as its input, for changing the excitation voltage and the input to AVR is added to the generator output. The components of conventional PSS shown in figure no.1-

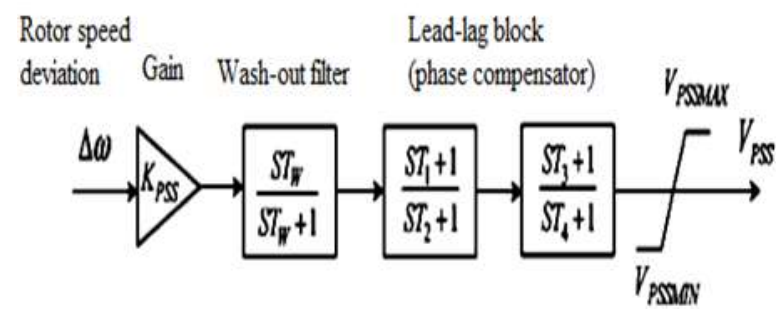

Figure 1 Structure of PSS

The main components of power system stabilizers are mainly gain, washout circuit, lead-lag compensator, torsional filter and limiters. [8]

The gain decides the amount of damping to th rotor oscillations. the damping increases with the increasing of gain upto certain limit after which damping reduces with the increment of gain value. The optimal PSS gain is chosen to damp the least damped mode. The optimal gain is related to the input speed of the stabilizer. The gain is selected of the damping factor below 0.05 . The high frequency gain sometimes reduces the effectiveness of the power system stabilizer. The high frequency sometimes becomes too high to lead noise amplification in the PSS operation. The choosing for gain value for PSS optimally is important.

For washout function Tw the time constant ranges from 1 to 20 seconds. It is used as high pass filter in the circuit. It removes the DC offset from the input signal. It also modifies the field voltage. For the local mode of oscillation $0.8-2.0 \mathrm{~Hz}$ the washout function takes a time of 1.5 seconds approximately. The washout time constant of 10 seconds or more is taken as desired value for inter-area oscillations. the value below 10seconds is not considerable at low frequency due to phase lead. If the phase lead is not compensated it will decrease the synchronizing component at inter-area oscillations. [9]

The lead-lag compensator improves an undesirable frequency response in the system at the feedback. PSS produces a torque in phase with rotor deviation for the damping of rotor oscillations. the phase lead compensates the lag between PSS output and the resultant electrical torque. And the phase lag is dependent upon the system conditions, generation parameters and types of exciters. A little amount of under compensation is preferred over overcompensation.

The torsional filter is used as low pass filter which is used to attenuate the first torsional mode of frequency. The torsional filter is used for adverse interaction of slip signal-based PSS with torsional oscillations. the torsional oscillation led the system to shaft damage. Even if the shaft damage not happened the output of the stabilizer can go to saturation for the presence of torsional frequency. The frequency range upto $3 \mathrm{~Hz}$ is minimized by the phase lag of torsional filter.

The function of the limiter in the PSS is to limit the generator terminal voltage during transient conditions. To prevent the PSS from performing the counter action of the AVR the limits of the generator terminal voltage is imposed in the design. The generator terminal voltage is set to 1.12-1.15 when there is high value of stabilizer output. During large swings in the machine there is a high-level positive output limit. The positive value of stabilizer is set to $0.1-0.2 \mathrm{pu}$.

\section{ROBUST CONTROL TECHNIQUES}

For developed countries PSS can be added to every generator in the system but the developing countries only a single PSS is added to highest unstable generator. The choice of single PSS in the system should be ideal to maintain the system stability. Nowadays, for the growing size of the utility the choice of the robust PSS is the best option compared to other types of PSS.

Robust controller design is required for gaining the robust stability which could be categorized by the disturbance rejection, guaranteed cost, robust poles localization, target sets etc. [10]

Ackerman's basic rules for robust control design are:

a) Robustness of control system choose from a range of physical activities and uncertainties of the model is not arbitrarily assumed.

b) Closing of the actuator constraints results slow system will perform slow and fast system will perform fast.

c) Be glum in analysis to assure the design.

The eigen value done to find the damping ratios, natural frequency, damping ratios of the system. The time domain analysis is performed to provide the rotor angle, field voltage, terminal voltage, torque, rotor speed for the system. The weighing functions should be chosen appropriately for the design. The loop shaping of the controller, frequency response and appropriate damping is provided by weighing factors. [12] 
The robust stabilization is made for different operating conditions which comprises the actual design of phase compensation and gain. The settling time, damping of oscillation, steady state error of robust PSS is reduced as compared to conventional PSS. The robust maintains the system stability by using the uncertainties in the system. [10]

The robust controller is designed by considering higher percentage worst case conditions. It can maintain the stability in higher percentage of sudden changes such as load conditions etc. The robust controllers are designed mainly by using the $\mathrm{H}$ infinity $(\mathrm{H} \infty)$ and $\mathrm{H} 2$ norm synthesis. The robust controllers maintain the Ricatti equations for the design. [11]

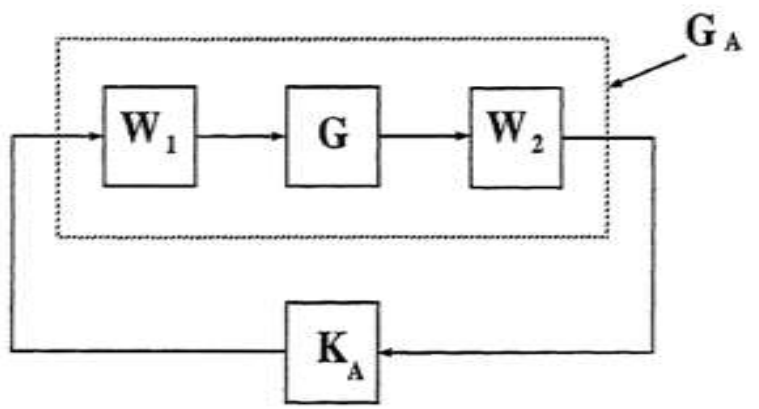

Figure 2 Shaped Plant

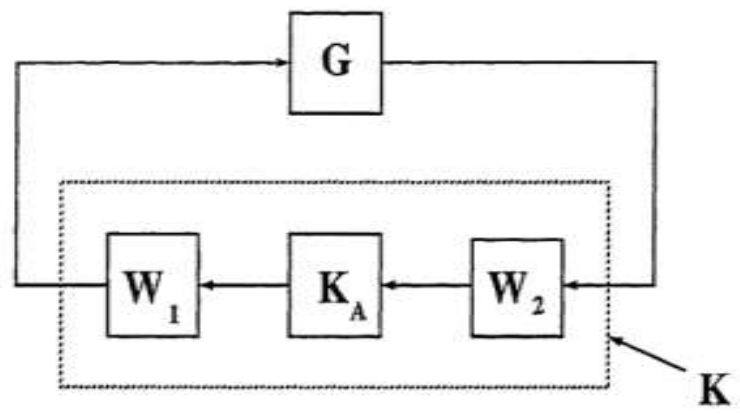

Figure 3 Final Controller

$\mathrm{W} 1$ is to be selected to keep the sensitivity $(\mathrm{S}=(\mathrm{I}+\mathrm{GK})-1)$ low at low frequencies such that $\mathrm{W} 1-1 \mathrm{~S} \leq 1$, while $\mathrm{W} 2$ is selected to keep the complimentary sensitivity $(\mathrm{T}=\mathrm{GK}(\mathrm{I}+\mathrm{GK})-1)$ low at high frequencies such that $\mathrm{W} 2-1 \mathrm{~T} \leq 1$. [6]

$\mathrm{W} 1$ and $\mathrm{W} 2$ are combined to form the loop shaped plant: $\mathrm{GA}=\mathrm{W} 2 * \mathrm{G} * \mathrm{~W} 1$

Whereas, the final feedback controller from figure 3 :

$\mathrm{K}=\mathrm{W} 1 * \mathrm{~K} \infty * \mathrm{~W} 2$

The pre-compensator acts as a lowpass filter for output disturbances or rejection and the post compensator as high pass filter to reduce the control effort in high frequency range.

\section{SINGLE MACHINE INFINITE BUS SYSTEM \& RESULTS}

The single machine connected to an infinite bus is the basic model to describe the small signal stability analysis. The single line diagram of a typical power system shown in figure 4 shows configuration for power evacuation from a remote power plant. The power from the plant is stepped up by the step-up generator and transmitted over a double-circuit transmission line to a large system, the terminal of which considered as an infinite bus. [7]

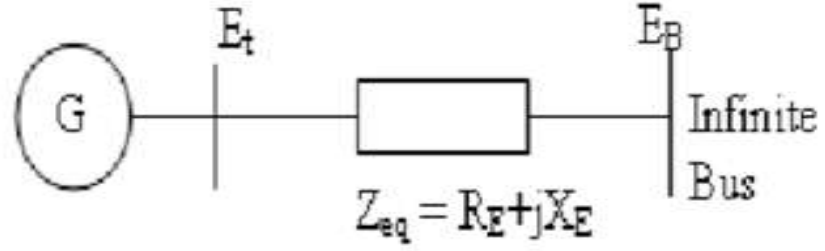

Figure 4 Single line diagram of SMIB

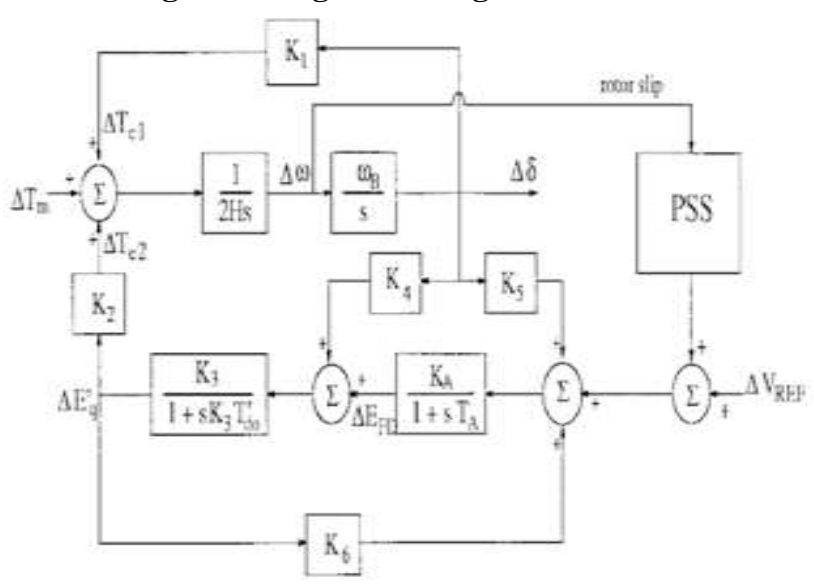

Figure 5 Block diagram representation of SMIB with PSS

The PSS is added to the SMIB as the feedback to the system. The PSS should have appropriate phase compensation circuits to compensate for phase lag between exciter input and generator electrical torque for the entire range of frequencies of rotor oscillations. This implies the phase characteristics of PSS should be exact inverse of composite exciter-generator phase characteristics. [16]

\section{SIMULATIONS AND RESULTS}

The simulation diagram shown in figure 6 shows the load angle, voltage magnitude deviation, accelerating torque, speed change and field voltage. The results are shown by varying the reference voltage and mechanical torque deviation. All the simulations are time domain analysis.

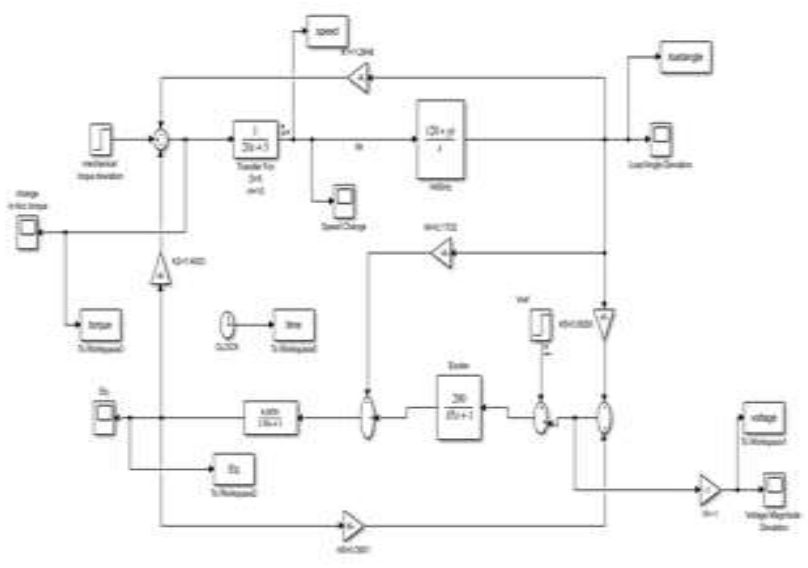

Figure 6 Simulation diagram of SMIB system

The subsystem of the conventional PSS provides the components is given in figure in figure 7 : 


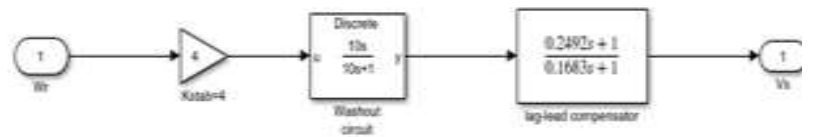

Figure 7 Block diagram of Conventional PSS

The robust PSS can be made from the results of the final $\mathrm{K}$ controller. The transfer function of the system is calculated:

$$
-48
$$

$$
\overline{s^{3}+20.77 s^{2}+268.3 s+65.8}
$$

The pre-compensator and post compensator values are chosen from the system to find the K controller.

The pre-compensator(W1) is calculated as:

$$
\frac{s+6.84}{s+0.02138}
$$

The post-compensator(W2) is calculated as:

$$
\frac{s+100}{s+200}
$$

From the $\mathrm{W} 1$ and $\mathrm{W} 2$ the transfer function of the final $\mathrm{K}$ controller is derived:

$$
\frac{1.287 e 06 s^{3}+4.678 e 07 s^{2}+4.175 e 08 s+1.895 e 09}{s^{4}+3.344 e 04 s^{3}+1.222 e 06 s^{2}+1.225 e 07 s+2.582 e 07}
$$

The transfer function is added to the block for robust PSS in the SMIB system as the feedback.

The different cases can be studied to compare the cases of without using PSS, using Conventional PSS and Robust PSS. The first case is considered as:

- Reference voltage is increased from 1-1.2pu at 60 seconds.

- Mechanical torque is increased from 1-1.2pu at 60seconds

The load angle for the system without PSS, with conventional PSS and Robust PSS are shown in figure 8, 9 and 10 respectively.

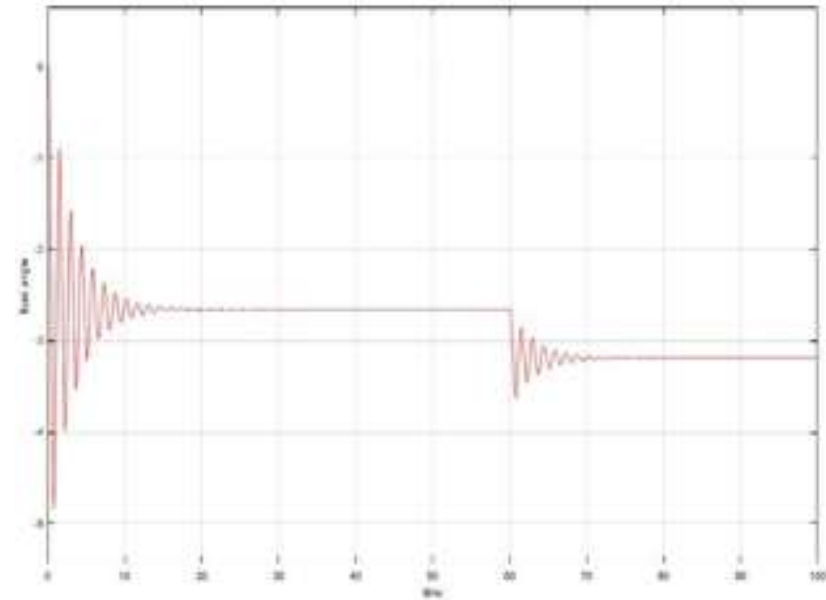

Figure 8 Load angle without PSS

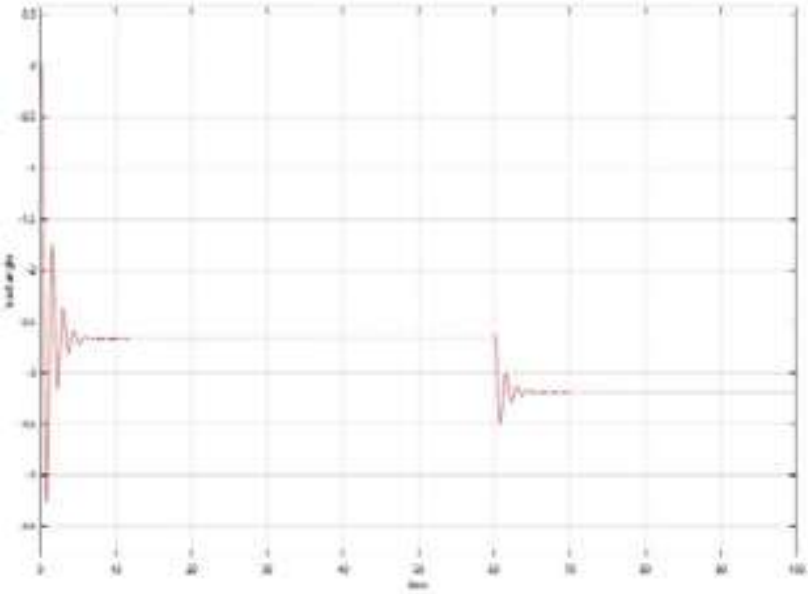

Figure 9 Load angle with Conventional PSS

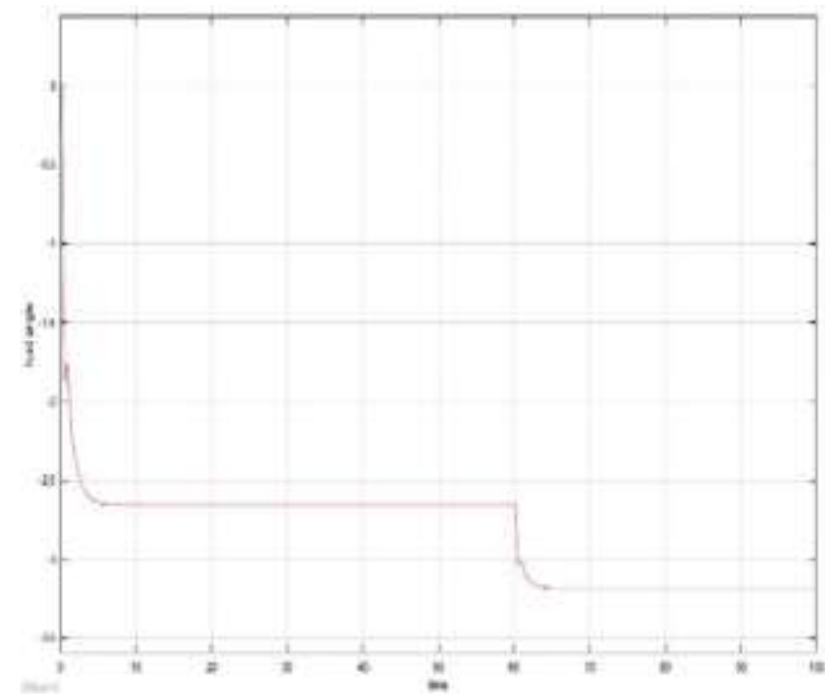

Figure 10 Load angle with Robust PSS

The speed change for the system without PSS, with conventional PSS and Robust PSS are shown in figure 11, 12 and 13 respectively.

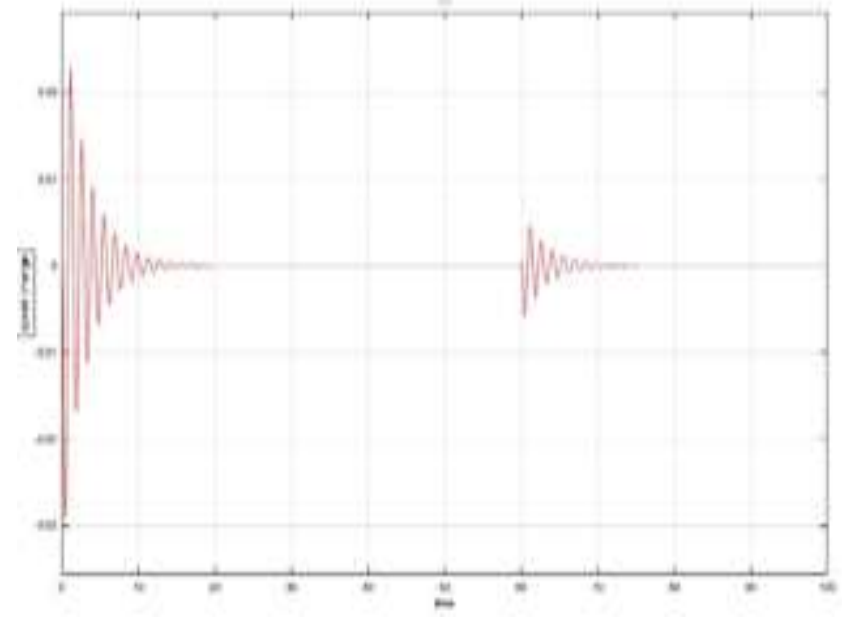

Figure 11 Speed Change without PSS

Published By: 


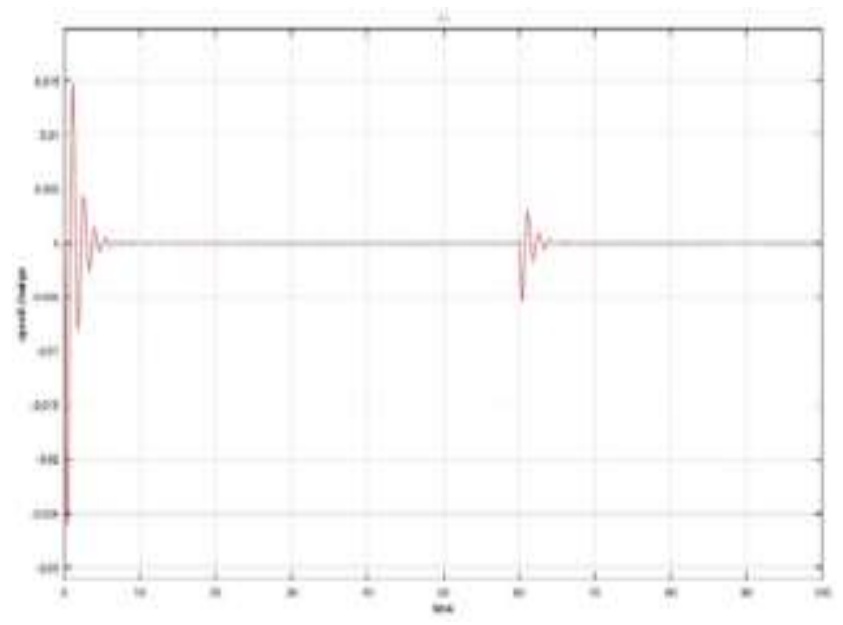

Figure 12 Speed Change with Conventional PSS

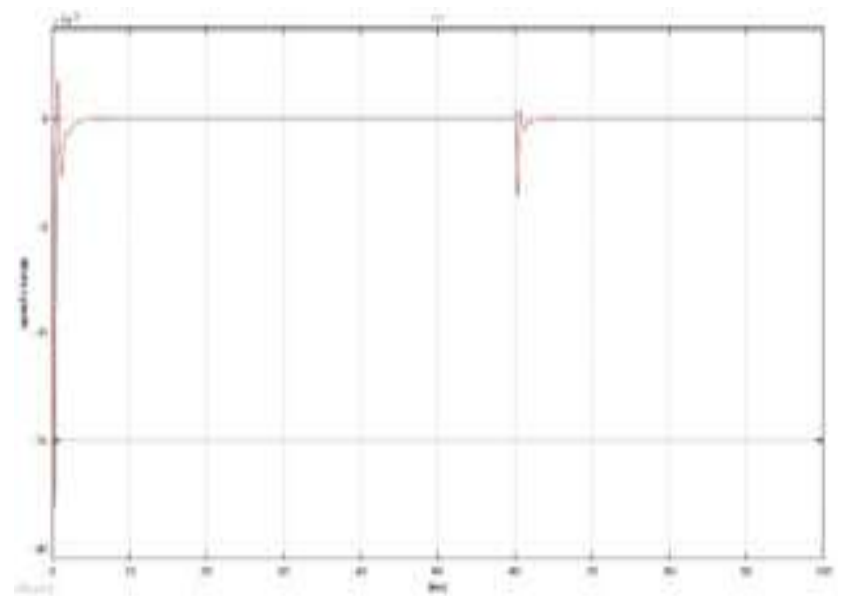

Figure 13 Speed Change with Robust PSS

The response of voltage magnitude for the system without PSS, with conventional PSS and Robust PSS are shown in figure 14,15 and 16 respectively.

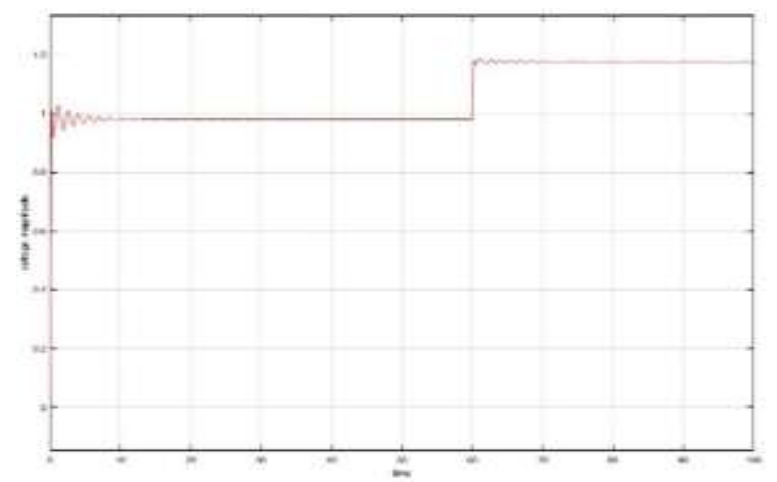

Figure 14 Voltage magnitude without PSS

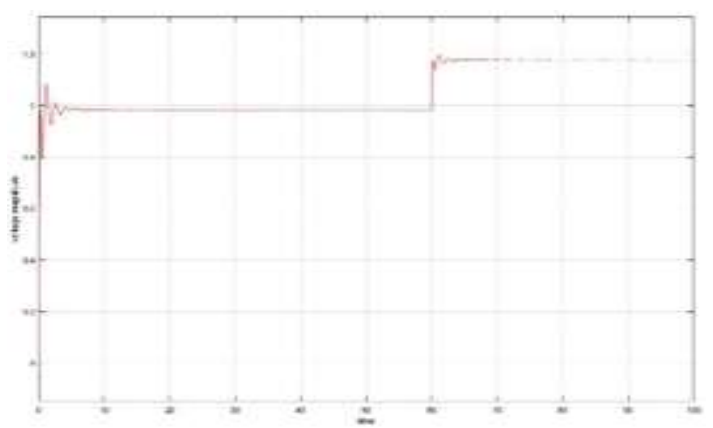

Figure 15 Voltage magnitude with Conventional PSS

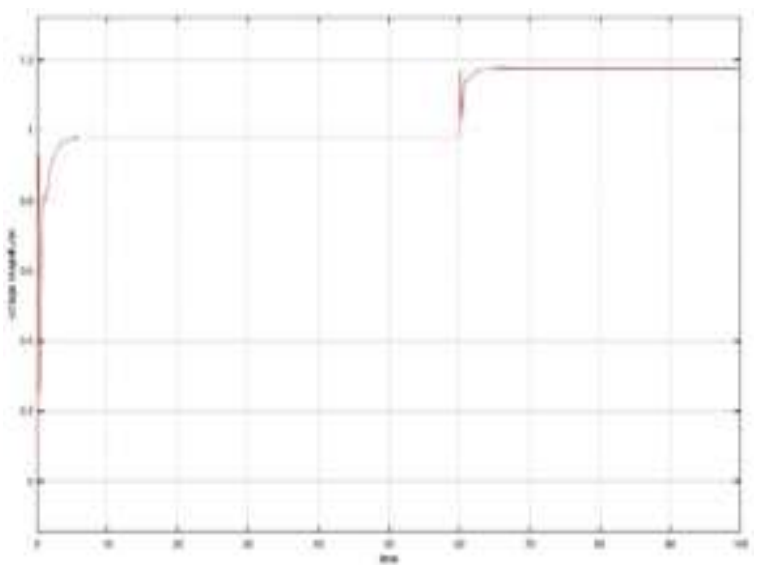

Figure 16 Voltage magnitude with Robust PSS

The second case is considered as:

Reference voltage is fixed at 1 pu for 60 seconds.

The mechanical torque is increased from 1-1.2pu. at 60 seconds.

The response of load angle for the system without PSS, with conventional PSS and Robust PSS are shown in figure 17,18 and 19 respectively.

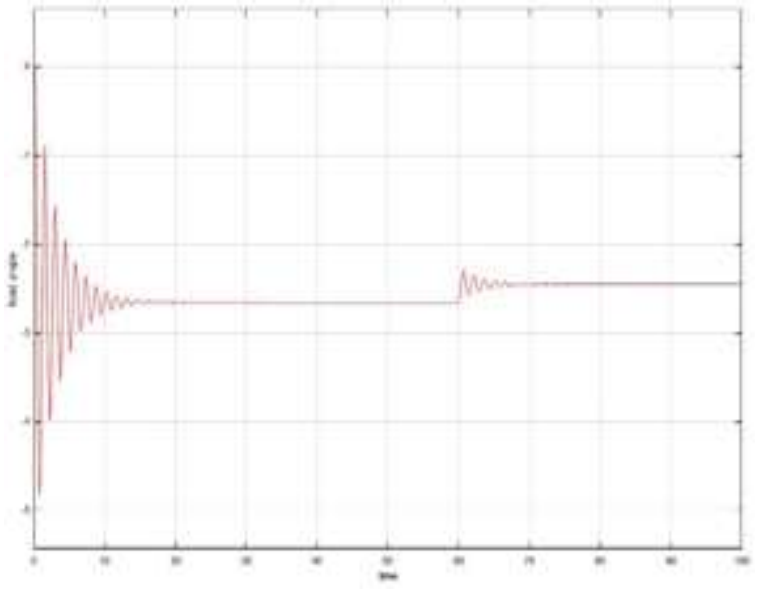

Figure 17 Load angle without PSS

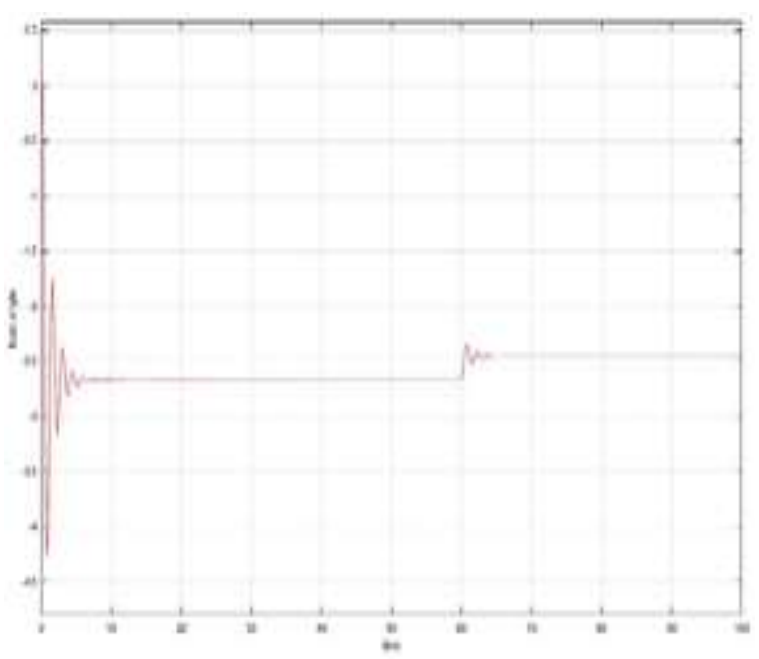

Figure 18 Load angle with Conventional PSS

Published By:

Blue Eyes Intelligence Engineering

\& Sciences Publication 


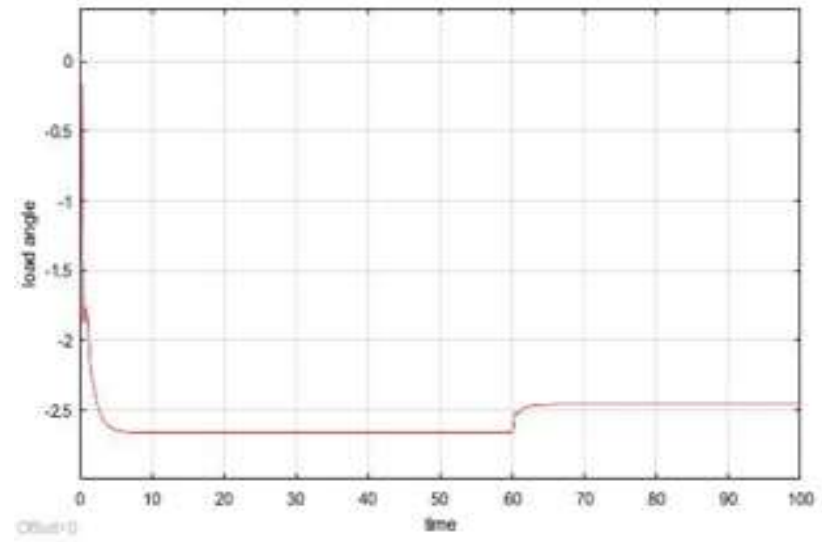

Figure 19 Load angle with Robust PSS

The speed change for the system without PSS, with conventional PSS and Robust PSS are shown in figure 20, 21 and 22 respectively.

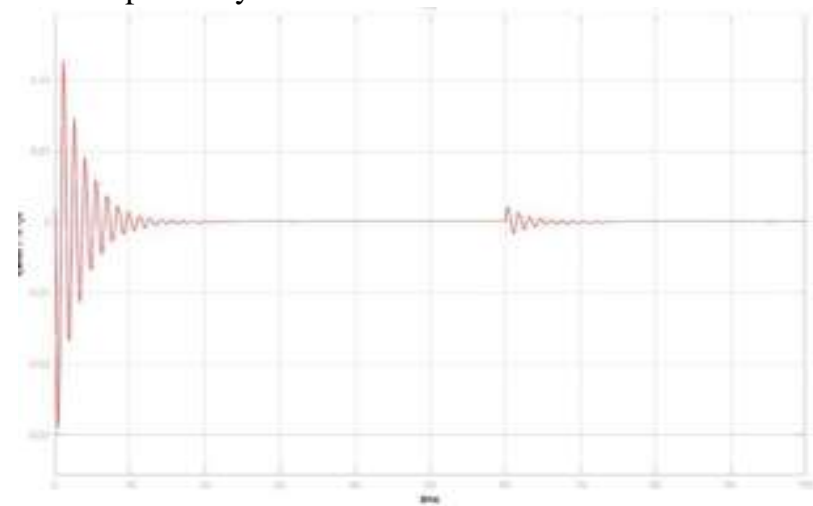

Figure 20 Speed Change without PSS

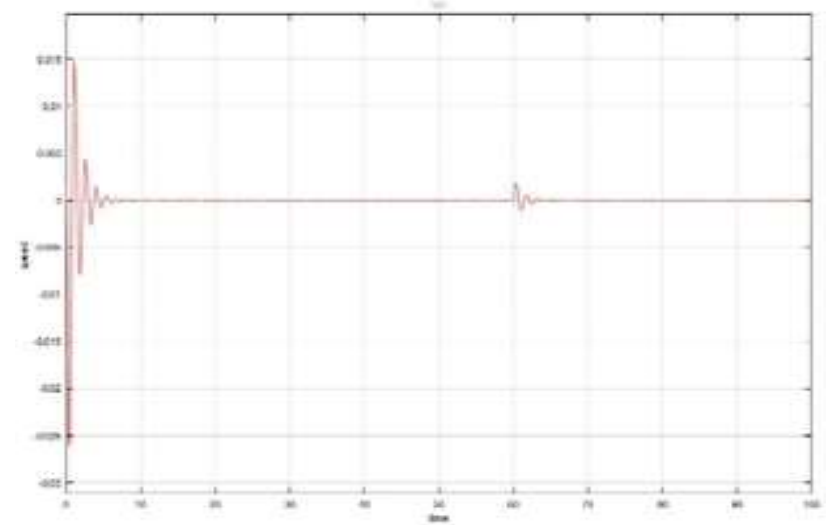

Figure 21 Speed Change with Robust PSS

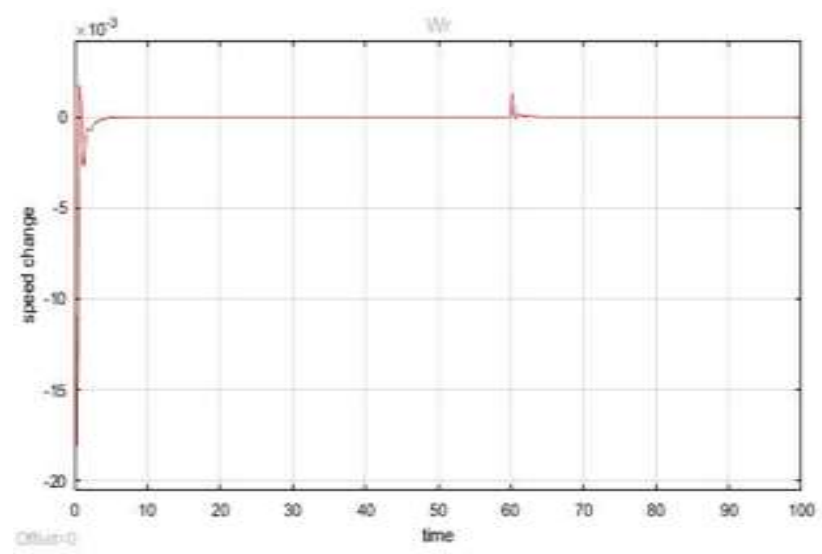

Figure 22 Speed change with Robust PSS
The response of voltage magnitude for the system without PSS, with conventional PSS and Robust PSS are shown in figure 23, 24 and 25 respectively.

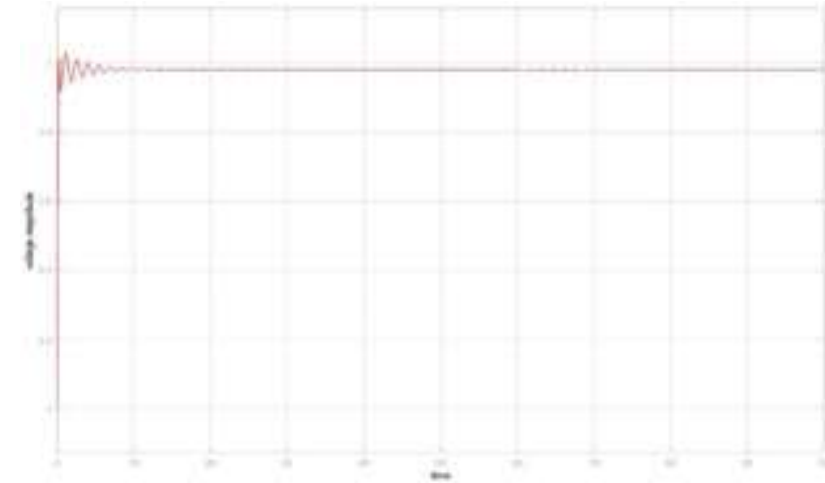

Figure 23 Voltage magnitude without PSS

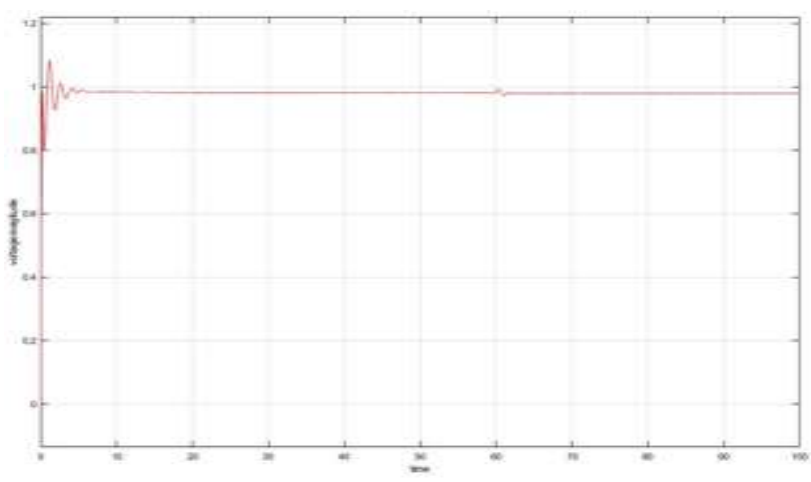

Figure 24 Voltage magnitude with Conventional PSS

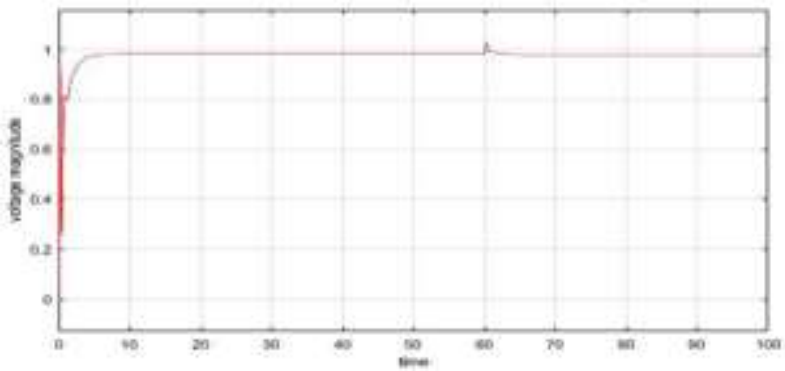

Figure 25 Voltage magnitude with Robust PSS

The third case is considered as:

- Mechanical torque is fixed at 1.0pu for 60 seconds

- Reference voltage is decreased from1-0.8pu at 30 seconds.

The response of load angle for the system without PSS, with conventional PSS and Robust PSS are shown in figure 26,27 and 28 respectively. 


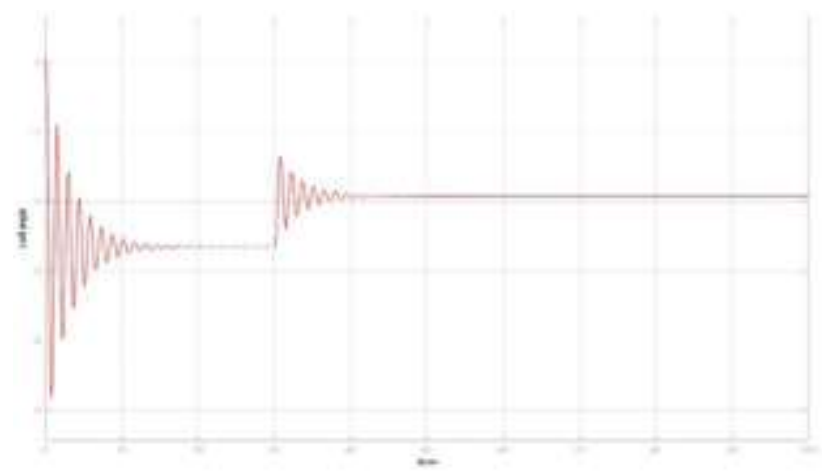

Figure 26 Load angle without PSS

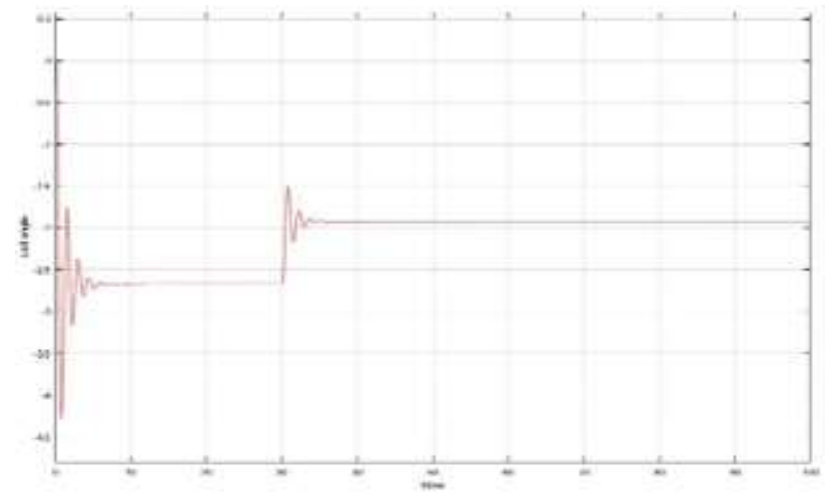

Figure 27 Load angle with Conventional PSS

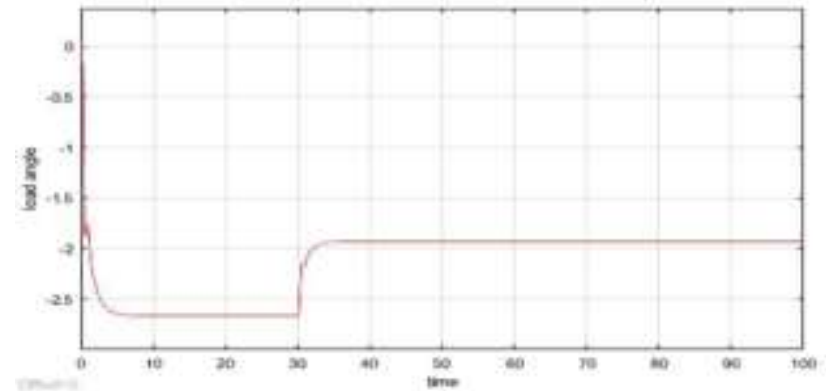

Figure 28 Load angle with Robust PSS

The speed change for the system without PSS, with conventional PSS and Robust PSS are shown in figure 29, 30 and 31 respectively.

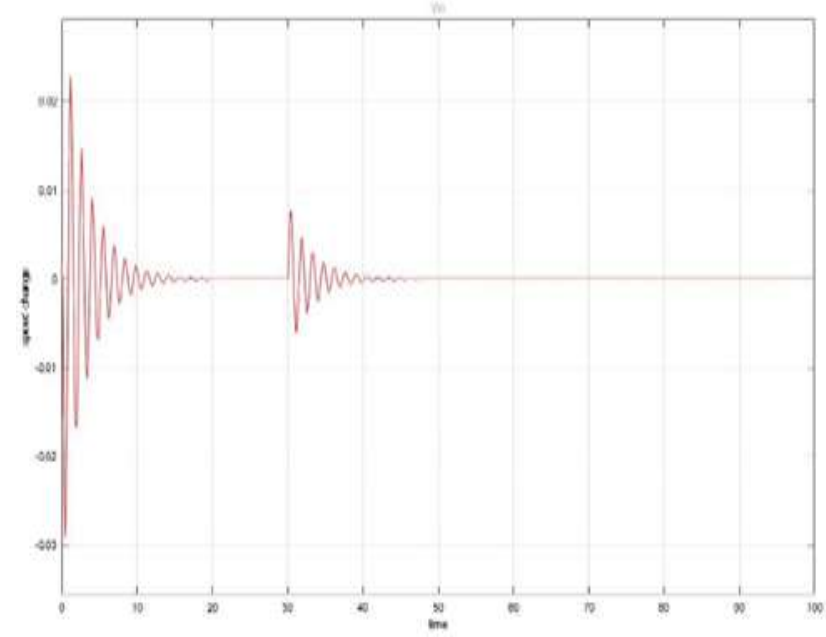

Figure 29 Speed change without PSS

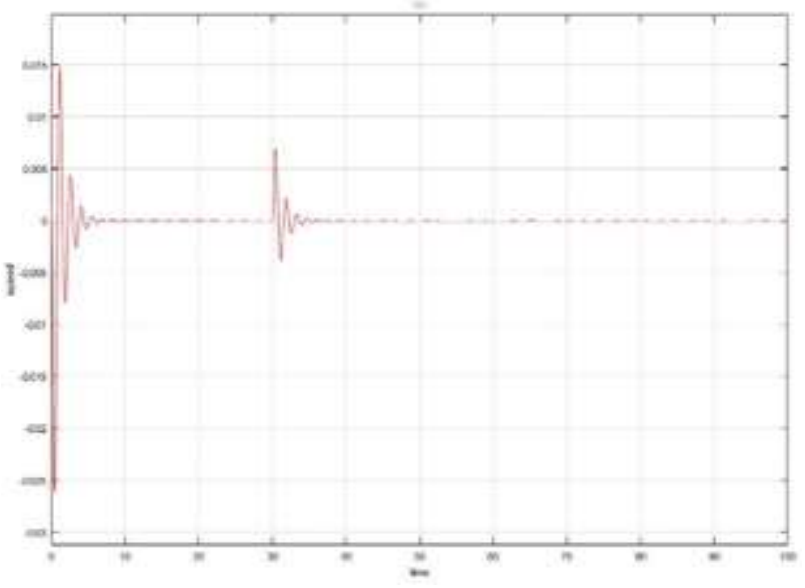

Figure 30 Speed Change with Conventional PSS

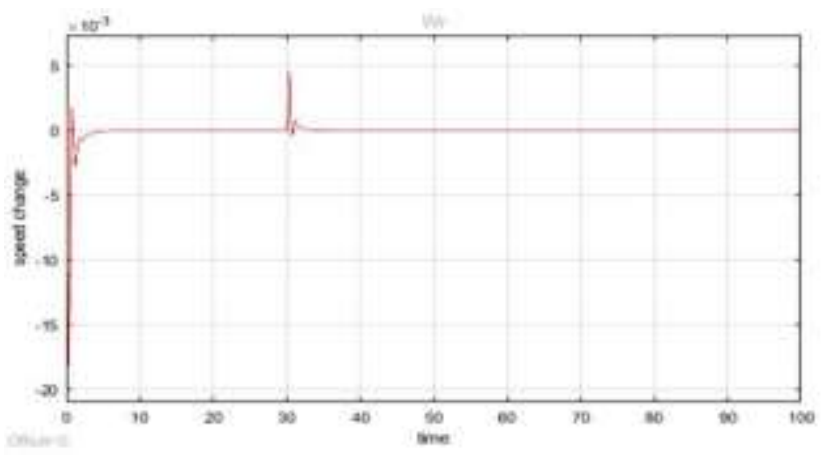

Figure 31 Speed change with Robust PSS

The response of voltage magnitude for the system without PSS, with conventional PSS and Robust PSS are shown in figure 32,33 and 34 respectively.

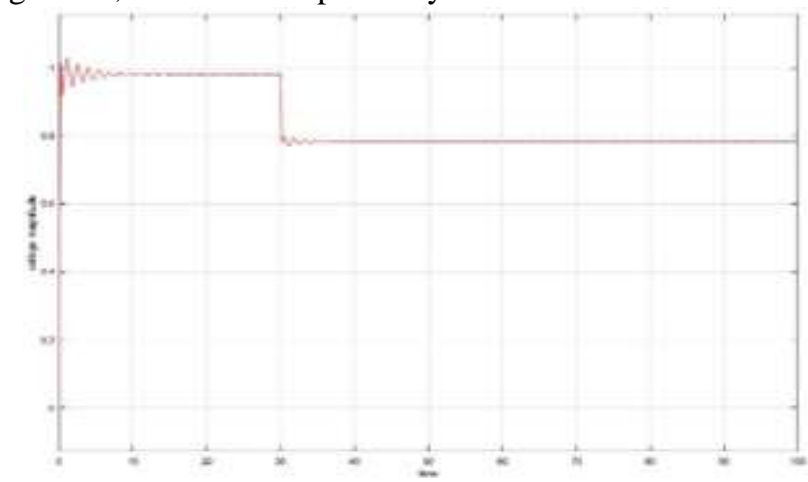

Figure 32 Voltage magnitude of without PSS

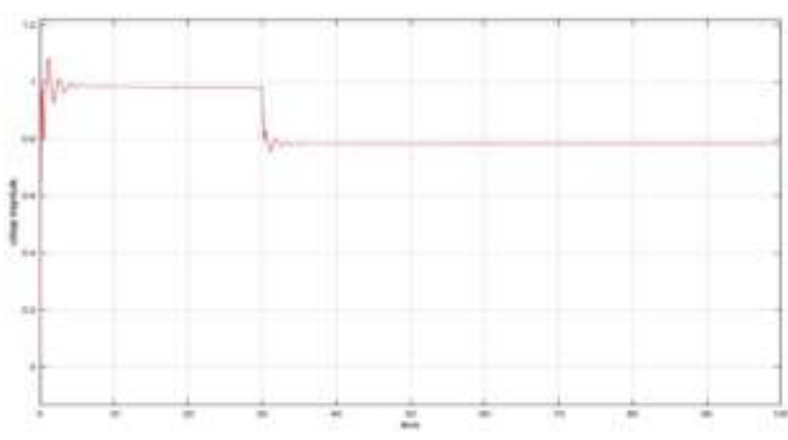

Figure 33 Voltage magnitude of Conventional PSS

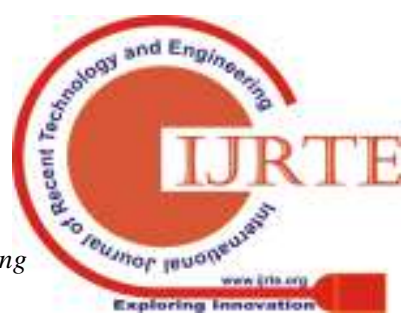




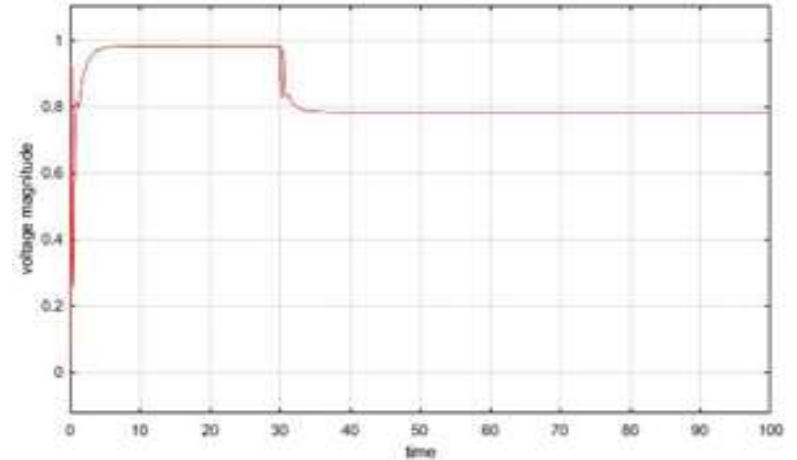

Figure 34 Voltage magnitude of Robust PSS

Comparison made for all the cases to show the effectiveness of the robust PSS to the system. System parameters like load angle, change in rotor speed and voltage magnitude deviation were observed. The addition of robust PSS to the system reduces the settling time and steady state errors for all the above cases.

\section{CONCLUSION}

The effective performance of the closed loop system was found to be satisfactory over a wide range of operating conditions making an appropriate and trade off between performance and voltage regulation by not changing the fundamental AVR concepts. The negative damping introduced by AVR is compensated with the PSS. Ease and flexibility of the design, to give a good feasible solution, are the main pros of the designed methodology.

\section{REFERENCES}

1. P. M. Anderson and A. A. Fouad, "Power System Control and Stability," John Wiley and sons, 2008.

2. P. Kundur, N. J. Balu and M. G. Lauby, "Power system stability and control," McGraw-Hill New York, 1994, Vol.7.

3. P. Kundur, M. Klein, G. Rogers and M. S. Zywno, "Application of power system stabilizers for enhancement of overall system stability," IEEE Transactions on Power Systems, Vol. 4, pp-614-626, 1989.

4. E. Larsen and D. Swann, "Applying power system stabilizer part i\&ii, Performance objectives and Tuning objects," IEEE transactions on Power Apparatus and systems, no.-6, 1981.

5. F. P. De Mello and C. Concordia, "Concepts of Synchronous Machine as Affected by Excitation Control", IEEE transaction on Power Apparatus and system, Vol. 88, No. 4, pp-316-328, April 1969.

6. K. Glover and D. McFarlane, "Robust Stabilization of normalized coprime factor plant descriptions with h-bounded uncertainty," IEEE Transactions on Automatic Control, Vol. 34, 1989.

7. R. Ramanujam, "Power System Dynamics Analysis and Simulation", Eatern Economy Edition, 2013.

8. K. Padiyar, "Power System Dynamics", B. S. Publications, 2008.

9. B. Pal and B. Chaudhuri, "Robust Control in Power Systems", Springer, 2006.

10. A. I. M. Ziad and M. M. Ali, "Robust Techniques for designing power system Stabilizer", Journal of Theoretical and Applied Information Technology.

11. V Sanjeeva Rao and Dr. B V Sanker Ram, "A Comprehensive Review of Power System Stabilizer Modeling, Analysis and Control Strategy", International Journal of Electrical and Electronics Engineers, Vol. No.
6, December 2014.

12. G. Naveen Kumar, Dr. M. Surya Kalavathi and B. Ravindranath Reddy, "Eigen Value Techniques for Small Signal Stability Analysis in Power System Stability", Journal of Theoretical and Applied Technology, January 2010.

13. Soumyabrata Barik and Dr. Abraham T Mathew, "Design and Comparison of Power System Stabilizer by Conventional and Robust H? Loop Shaping Technique", International Conference on Circuit Power and Computing Technologies, 2014.

14. Dushyant Sharma and Sukumar Mishra, "Power System Frequency Stabilizer for Modern Power Systems", IET Generation, Transmission and Distribution, January 2018.

15. P. Kundur, G. J. Rogers, D. Y. Wong, L.Wang and M. G. Lauby, "A compressive Computer Program Package for Small Signal Stability Analysis of Power Systems", IEEE Transactions on Power Systems, Vol. 5, No. 4, November 1990.

16. Jyapal.R and Dr. J. K. Mendiratta, "H? Controller Design for a SMIB Based PSS Model 1.1", Journal of Theoretical and Applied Information Technology, January 2010.

17. John F. Hauer, "Robust Damping Controls for Large Power systems", IEEE control Systems Magazine, February 1989.

18. H. M. Solinman, A. L. Elsafei, A. A. Shaltout and M. F. Morsri, "Robust Power Stabilizer", IEEE Transactions on Power Systems, 1998. 